Monte Carlo dosimetry for forthcoming clinical trials in x-ray microbeam radiation therapy

This article has been downloaded from IOPscience. Please scroll down to see the full text article.

2010 Phys. Med. Biol. 554375

(http://iopscience.iop.org/0031-9155/55/15/012)

View the table of contents for this issue, or go to the journal homepage for more

Download details:

IP Address: 147.83.126.225

The article was downloaded on $28 / 07 / 2010$ at $11: 48$

Please note that terms and conditions apply. 


\title{
Monte Carlo dosimetry for forthcoming clinical trials in $x$-ray microbeam radiation therapy
}

\author{
I Martínez-Rovira ${ }^{1,2}, \mathbf{J}$ Sempau $^{2}, \mathbf{J}$ M Fernández-Varea ${ }^{3}$, A Bravin ${ }^{1}$ \\ and Y Prezado ${ }^{1}$ \\ ${ }^{1}$ ID17 Biomedical Beamline, European Synchrotron Radiation Facility (ESRF), B.P. 220, \\ 6 Jules Horowitz, F-38043 Grenoble Cedex, France \\ 2 Institut de Tècniques Energètiques, Universitat Politècnica de Catalunya, Diagonal 647, \\ E-08028 Barcelona, Spain \\ ${ }^{3}$ Facultat de Física (ECM and ICC), Universitat de Barcelona, Diagonal 647, \\ E-08028 Barcelona, Spain \\ E-mail: yolanda.prezado@esrf.fr
}

Received 6 November 2009, in final form 4 June 2010

Published 20 July 2010

Online at stacks.iop.org/PMB/55/4375

\begin{abstract}
The purpose of this work is to define safe irradiation protocols in microbeam radiation therapy. The intense synchrotron-generated $\mathrm{x}$-ray beam used for the treatment is collimated and delivered in an array of $50 \mu \mathrm{m}$-sized rectangular fields with a centre-to-centre distance between microplanes of $400 \mu \mathrm{m}$. The absorbed doses received by the tumour and the healthy tissues in a human head phantom have been assessed by means of Monte Carlo simulations. The identification of safe dose limits is carried out by evaluating the maximum peak and valley doses achievable in the tumour while keeping the valley doses in the healthy tissues under tolerances. As the skull receives a significant fraction of the dose, the dose limits are referred to this tissue. Dose distributions with high spatial resolution are presented for various tumour positions, skull thicknesses and interbeam separations. Considering a unidirectional irradiation (field size of $2 \times 2 \mathrm{~cm}^{2}$ ) and a centrally located tumour, the largest peak and valley doses achievable in the tumour are $55 \mathrm{~Gy}$ and $2.6 \mathrm{~Gy}$, respectively. The corresponding maximum valley doses received by the skin, bone and healthy brain are $4 \mathrm{~Gy}$, $14 \mathrm{~Gy}$ and $7 \mathrm{~Gy}$ (doses in one fraction), respectively, i.e. within tolerances (5\% probability of complication within 5 years).
\end{abstract}

(Some figures in this article are in colour only in the electronic version)

\section{Introduction}

Gliomas are extremely radioresistant tumours. The high absorbed doses needed to ablate gliomas are limited by the high morbidity of the surrounding healthy tissue, especially 
in children (Kagan et al 1976). The average lifespan for these patients is less than 1 year, and generally no patient survives 5 years after treatment (Behin et al 2003). Highgrade gliomas are still of poor prognostic value despite the development of many innovative therapies. Stereotactic radiosurgery (Phillips et al 1994), intensity-modulated radiation therapy (Cardinale et al 1998) and boron neutron capture therapy (Barth et al 2005) are some examples. The use of radiotherapy plus concomitant and adjuvant temozolomide has allowed a significant prolongation of survival (Stupp et al 2002, 2005, 2009). However, the outcome still remains unsatisfactory and the management of glioblastomas is mainly palliative.

One possible way to improve the therapeutic index in radiotherapy is to employ new techniques based on what is known as the dose-volume effect: the smaller the field size, the higher the tolerance of the healthy tissue (Curtis 1967). Microbeam radiation therapy (MRT) is a synchrotron radiotherapy technique that relies on this fact. Such an irradiation can be palliative or curative while causing minimal damage to the contiguous healthy tissue.

In MRT, the irradiation is carried out by means of an array of parallel x-ray microbeams (from 25 to $50 \mu \mathrm{m}$ thick), with a centre-to-centre (c-t-c) distance between microbeams of 200 or $400 \mu \mathrm{m}$. The x-ray energy spectrum ranges from about 50 to $500 \mathrm{keV}$, with a mean energy around $100 \mathrm{keV}$ (Siegbahn et al 2006). The microbeams are produced by a multi-slit collimator that spatially fractionates in the horizontal direction the beam coming from the synchrotron source (Bräuer-Krisch et al 2009). Targets are then vertically scanned through the microfractionated beam to deliver microplanes of $x$-rays. The synchrotron origin of the microbeams confers them two crucial features: negligible divergence (allowing the production of sharply defined beam edges in tissue) and high flux (enabling a fast irradiation process that prevents motion artifacts of the subject caused by cardiosynchronous pulsation).

The MRT irradiation scheme results in dose profiles consisting of a pattern of peaks and valleys, i.e. with high doses in the microbeam paths and low doses in the spaces between them (Siegbahn et al 2006). The minimum dose in the central region between two microbeams is named valley dose and the dose in the centre of the microbeam is the peak dose.

The ratio between the peak dose and the valley dose (peak-to-valley dose ratio, PVDR) depends on the incident $x$-ray beam energy, the tissue composition, the beam thickness, the c-t-c distance and the irradiation field size but it does not depend on the peak dose prescription in the tumour. Despite being an important dosimetric parameter in this type of spatially fractionated techniques, PVDR is a relative quantity and it is also necessary to have the information on both peak and valley doses.

During the last two decades, several preclinical studies have shown the sparing effect of the healthy tissue using MRT on the brain of adult rats (Slatkin et al 1995, Regnard et al 2008), suckling rats (Laissue et al 1999), duck embryos (Dilmanian et al 2001), piglets (Laissue et al 2001), chick-embryo chorio-amniotic membranes (Blattmann et al 2005) and nude mice (Serduc et al 2008). In parallel, it has been proven that MRT can ablate highly aggressive animal tumour models such as 9L brain gliomas (Laissue et al 1998, Dilmanian et al 2002, Smilowitz et al 2006), EMT-6 carcinoma (Dilmanian et al 2003) and SCCVII carcinoma (Miura et al 2006).

Dilmanian et al (2002) stressed the fact that MRT could cure 9L-bearing animals without killing all tumoral cells. They suggested that MRT effects might involve mechanisms other than a direct ionizing radiation effect on tumoral cells, like poor regenerative capacity of tumoral vessels after radiation exposure. Additional mechanisms such as abscopal effects may also play a role. The analysis of the results for different beam spacings from Dilmanian et al (2002) and Regnard et al (2008) suggests that a high lesion density induced by the 
microbeams on tumour vasculature could be sufficient to treat brain tumours. Indeed, a decrease in microbeam spacing may increase the number of vascular lesions per unit volume.

Nevertheless, the biological mechanisms following an MRT irradiation are not yet fully understood. It has been hypothesized that the sparing effect in the healthy tissue along the beam paths is due to a rapid biological repair of the microscopic lesions by the minimally irradiated cells contiguous to the irradiated tissue slices (Slatkin et al 1995, Laissue et al 1998, Dilmanian et al 2001). Dilmanian et al (2002) have shown that the sparing effect of MRT seems to depend mostly on the valley dose. The brain-sparing effect (measured by the onset of the appearance of white matter necrosis) vanishes only when the valley dose approaches the tissue tolerance to broad beams. As for the PVDRs, the valley doses depend on the X-ray beam energy spectrum, on the spacing between microbeams, on the irradiation field size but also on the peak doses. A smaller c-t-c distance results in higher valley doses and in lower tolerances (Dilmanian et al 2002, Regnard et al 2008).

Following the success of preclinical studies, the Biomedical Beamline ID17 of the European Synchrotron Radiation Facility (ESRF) is planning to proceed towards clinical trials (Laissue et al 2007, Renier et al 2008). In this context, Monte Carlo (MC) absorbed dose calculations in realistic phantoms are needed to define irradiation protocols in MRT.

The first MC dosimetry studies in MRT were done by Slatkin et al (1992). The simulations were performed for $25 \mu \mathrm{m}$ thick microbeams spaced 50, 100 or $200 \mu \mathrm{m}$, impinging on a $16 \mathrm{~cm}$ long cylindrical water phantom with a diameter of $16 \mathrm{~cm}$. Subsequent works considered similar water phantoms (Stepanek et al 2000, De Felici et al 2005, Siegbahn et al 2006, Spiga et al 2007). Company and Allen (1998) calculated also the dose distribution in a tissue/lung/ tissue phantom and Orion et al (2000) in a spherical human head phantom with a diameter equal to $17.2 \mathrm{~cm}(0.6 \mathrm{~cm}$ of skull and $16 \mathrm{~cm}$ of brain). Prezado et al (2009b), who investigated the dose enhancement in MRT by loading the tumour with gadolinium, have also performed the simulations in a human head phantom inspired by the one described in the work of Harling et al (1995).

It is important to point out that the use of realistic geometries is essential for a correct dose assessment. For example, the valley dose in a centrally located tumour in a water phantom is around 2.8 times higher than that of the head phantom considered in the present study. The difference has to be mainly ascribed to the presence of the bone.

To the best of our knowledge, the only dosimetric studies carried out in MRT using a head phantom are those of Orion et al (2000) and Prezado et al (2009b). Orion et al (2000) used $30 \mu \mathrm{m}$ thick microbeams, a c-t-c distance between microplanes of 100 and $200 \mu \mathrm{m}$ and field sizes of $3 \times 3$ and $6 \times 6 \mathrm{~cm}^{2}$. In Prezado et al $(2009 \mathrm{~b})$, the microbeam width, the c-t-c distance and the irradiation field size were $50 \mu \mathrm{m}, 200 \mu \mathrm{m}$ and $2 \times 2 \mathrm{~cm}^{2}$, respectively. The PVDR value for a lateral irradiation of a centrally located tumour in Prezado et al (2009b) is in agreement with our results in section 3.4 and in figure 6.

None of the parameters used in previous papers are going to be employed in the forthcoming clinical trials at the ESRF. The chosen irradiation parameters have been fixed to a microbeam width of $50 \mu \mathrm{m}$ and a c-t-c distance of $400 \mu \mathrm{m}$ in order to reach a compromise between healthy tissue sparing and tumour eradication.

For the first time, this work presents a systematic study of the dose distribution and the corresponding conversion of absorbed dose to normalized total dose considering biological effects in a realistic head phantom with a high spatial resolution. The maximum dose prescription achievable in the tumour is evaluated in different cases by adopting the criterion of keeping the valley dose in the healthy tissue as low as possible and below tissue tolerances for a broad beam (Dilmanian et al 2002). In this way, safe and conservative irradiation protocols in MRT can be defined. 


\section{Materials and methods}

\subsection{Monte Carlo simulation code: PENELOPE/penEasy}

The MC code PENELOPE (Salvat et al 2006, Sempau et al 1997) has been employed. PENELOPE simulates the coupled transport of photons, electrons and positrons in the energy interval from $50 \mathrm{eV}$ to $1 \mathrm{GeV}$, and in arbitrary material systems. PENELOPE has been widely used in the medical physics field, see for example Sempau et al (2001), Torres et al (2004), Sempau and Andreo (2006), Fernández-Varea et al (2007) and Sterpin et al (2008) to name a few. In this work, penEasy (Sempau and Badal 2008), a structured general-purpose main program for PENELOPE, has been adopted to do the calculations.

The most relevant photon interaction mechanisms in the energy range of concern in MRT (mean energy $\sim 100 \mathrm{keV}$ ) are Compton scattering and photoelectric effect. These interactions are simulated one by one until the photon reaches an energy lower than a userdefined threshold (the absorption energy). Compton scattering is modelled by means of the relativistic impulse approximation. This formalism takes into account both Doppler broadening and binding effects of the atomic electrons. Photoelectric cross sections are interpolated from the tabulations of the Evaluated Photon Data Library (Cullen et al 1997). Many-body and aggregation effects are ignored and atoms are considered as independent.

Regarding electron transport, PENELOPE implements a mixed simulation algorithm in which interactions are classified into hard and soft. They are regarded as hard when the energy loss or the angular deflection of the transported particle is above certain user-defined cutoffs. Hard interactions are simulated individually. Soft interactions are simulated by resorting to multiple-scattering theories. Angular deflections are mainly caused by elastic scattering, while inelastic collisions are the dominant energy loss mechanism. Elastic scattering cross sections are calculated from relativistic partial-wave analysis (Salvat et al 2005). Inelastic scattering is described by means of a schematic generalized oscillator strength model with mean excitation energy values taken from the ICRU Report 37 (ICRU 1984).

\subsection{Simulation geometry and configuration}

The considered head phantom is composed of slabs of skin, bone and brain, with thicknesses equal to $2 \mathrm{~mm}, 1 \mathrm{~cm}$ and $16 \mathrm{~cm}$, respectively. The dimensions of the human brain are taken from Harling et al (1995).

To obtain the dose distribution for the whole field, we have adopted the following approach. First, we compute the dose distribution for a single microbeam in the head phantom. Next, for each spatial location, the dose is evaluated as the sum of the contributions of each individual microbeam to cover the desired irradiation field. In agreement with previous dosimetry works (Boudou et al 2005, Prezado et al 2009a, Prezado et al 2009b), a field size of $2 \times 2 \mathrm{~cm}^{2}$ is assumed in the simulations, which corresponds to the tumour size. In the calculations, the dose prescription is referred to as the centre of a 'virtual' tumour located at a certain depth.

Absorbed doses are tallied in voxels of $2 \mu \mathrm{m}$ in the lateral direction of the microbeam, $1 \mathrm{~mm}$ in the vertical direction of the microbeam and $2 \mathrm{~mm}$ in depth. Doses were scored up to $2 \mathrm{~cm}$ away from the centre of the microbeam in the lateral direction (see figure 1).

There are different simulation parameters to be considered in PENELOPE. Users are required to define an absorption energy (EABS) for each particle type $\left(\gamma, \mathrm{e}^{ \pm}\right)$at which histories are terminated and their remaining energy is locally absorbed. Charged-particle transport also requires the setting of the following parameters: $\mathrm{C} 1$, which determines the average angular deflection between two consecutive hard elastic events; $\mathrm{C} 2$, which defines the maximum average fractional energy loss in a single multiple-scattering step; WCC, the cutoff energy for 


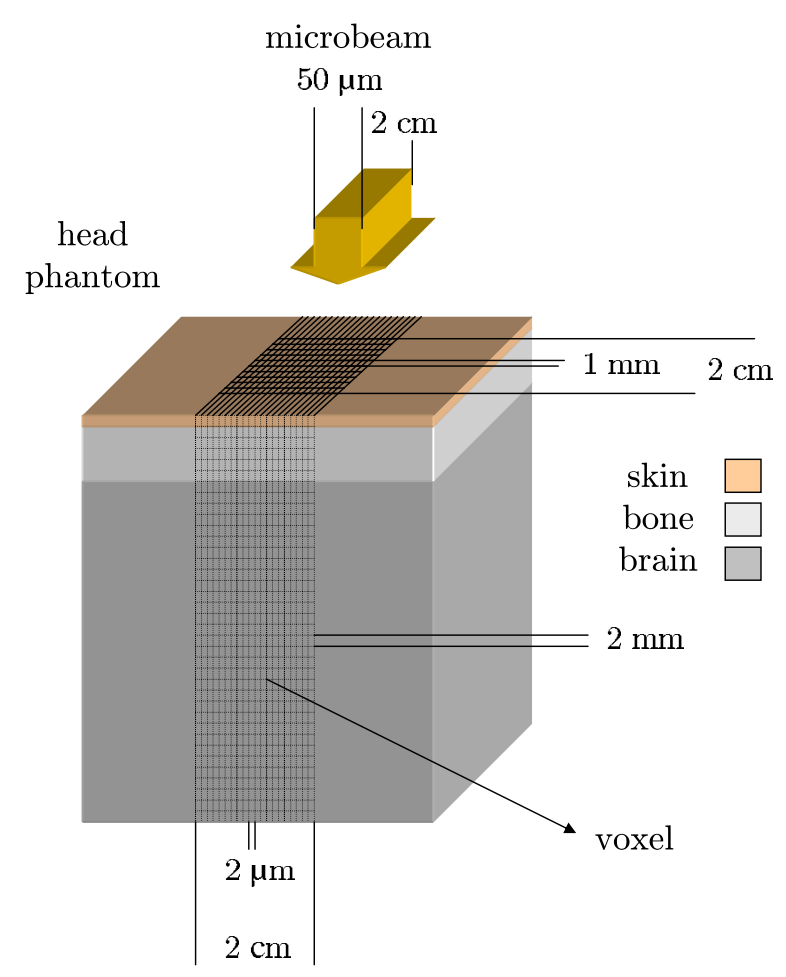

Figure 1. Scheme of the dimensions and orientation of the head phantom, as well as the voxels used to tally the dose distribution in the simulations (not to scale).

the production of hard inelastic events; WCR, the cutoff energy for the production of hard bremsstrahlung; and DSMAX, the maximum allowed step length.

In our simulations, EABS is set to $300 \mathrm{eV}$ for all particles, to ensure that the photon mean free path and the secondary-electron range are smaller than the minimum bin width used to tally the dose distributions. $\mathrm{C} 1$ and $\mathrm{C} 2$ are set to 0.01 , which is a very conservative value. WCC and WCR are set equal to EABS. Finally, the value of DSMAX is chosen to be one tenth of the different slab thicknesses.

Simulations are discontinued when the average statistical uncertainty is less than $0.5 \%$ (2 standard deviations). This uncertainty is calculated as an average of the uncertainties of the bins with doses above half of the maximum score.

\subsection{Dose equivalence with standard fractionation schemes}

Since in MRT the doses are delivered in one fraction, the expected biological effects and tissue tolerances will depart from the values in conventional radiotherapy, where the doses are typically delivered in $2 \mathrm{~Gy} /$ session (Emami et al 1991, QUANTEC 2010). To establish the equivalence of the valley dose with the standard fractionation scheme, the normalized total dose (NTD) has been evaluated from

$$
\mathrm{NTD}_{2.0}=n d\left(1+\frac{d}{\alpha / \beta}\right)\left(1+\frac{2 \mathrm{~Gy}}{\alpha / \beta}\right)^{-1},
$$

where $\mathrm{NTD}_{2.0}$ is the NTD corresponding to the standard fractionation scheme of $2 \mathrm{~Gy} /$ fraction, $d$ is the absorbed dose per fraction and $n$ is the number of sessions. In our case, $n=1$ and the 
total dose given in one MRT fraction is $D=n d=d$. The $\alpha / \beta$ ratio is a parameter related to the biological response of the tissue under consideration and depends on its renewal capability.

Equation (1) is derived from the linear-quadratic (LQ) model (Flickinger and Kalend 1990) for the biological response to ionizing radiation, often employed to compare different fractionation schemes in conventional radiotherapy because it fits the experimental cell survival curves quite well in most cases. It is widely used to quantify the effects of radiotherapy at low and medium doses, but it might overestimate the magnitude of cell killing for doses greater than 10 Gy (Brenner 2008). Several attempts have been made to extend the LQ model to higher doses per fraction, all of them leading to the inclusion of at least one additional parameter in the formalism (Lind et al 2003, Guerrero and Li 2004). Unfortunately, the applicability is limited because most clinical datasets are insufficient to estimate all the parameters (Joiner and van der Kogel 2009).

There are ongoing discussions in the scientific community on the applicability of the LQ model at high doses per fraction due to the lack of clinical data (Kirkpatrick et al 2009). In vivo studies have suggested that the predictions of the LQ model are still acceptable for the design of clinical trials based on doses per fraction up to $18 \mathrm{~Gy}$ (Brenner 2008). In vitro investigations have shown that the cell survival followed the standard LQ model up to 15 Gy (Garcia et al 2006). Theoretical works indicate that the LQ model remains valid up to $17 \mathrm{~Gy}$ (Sachs et al 1997). As the valley dose in the present work ranges from 1 to 14 Gy in one fraction, equation (1) can be used with reasonable confidence.

The values of $\alpha / \beta$ range from 8 to 15 Gy (Steel 2002) for tumours and early responding tissues. The skin is a rapidly renewable tissue for which $\alpha / \beta=8.8 \mathrm{~Gy}$ (Turesson and Thames 1989). For late responding tissues such as the brain and the bone, the values of $\alpha / \beta$ are smaller: 2 Gy (Steel et al 2002) and 1.8 Gy (Overgaard 1988), respectively. In the latter cases, the dose fractionation effect and the repair mechanisms between consecutive fractions are more important than in early responding tissues, where the total absorbed dose plays a more significant role.

The valley dose is converted into $\mathrm{NTD}_{2.0}$ to assess if it is under the tolerance level for the well-established conventional radiotherapy limits, following the results by Dilmanian et al (2002). According to the latest published compilations of values on human patients, the tolerance doses (with a 5\% probability of complication within 5 years from treatment, irradiation of one-third of the whole organ volume) are 70 Gy for the skin and 50-60 Gy for the bone (Emami et al 1991). In the case of the brain, a 5\% risk of symptomatic radiation necrosis with the standard fractionation scheme is predicted to occur at 72 Gy (Lawrence et al 2010). For single fractionation radiosurgery, a clear correlation has been demonstrated between the target size and the risk of adverse events. For targets smaller than $20 \mathrm{~mm}$ in diameter, the maximum brain tolerance dose is higher than 24 Gy (Shaw et al 2000).

Emami et al (1991) also present the tolerance doses for $50 \%$ probability of necrosis within 5 years: $>70$ Gy for the skin and 65-75 Gy for the brain and the bone. The peak dose relative to this latter case has been studied as well.

\section{Results and discussion}

Dose distributions for various positions of a tumour, skull thicknesses and interbeam separations have been computed. The influence of these variables on the dose distributions will be presented separately. Dose is reported as absorbed dose to medium.

The valley dose presented in the figures that follow is obtained in the centre of the field since this is the location of the largest valley dose. A conservative criterion is to consider that the absorbed dose in this valley must be lower than the tolerances in conventional radiotherapy. 


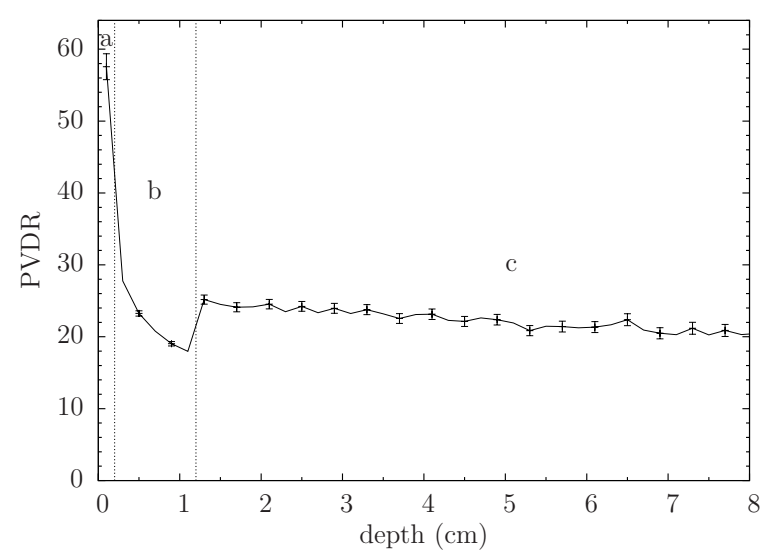

Figure 2. PVDR as a function of depth in the head phantom considering a unidirectional irradiation with $50 \mu \mathrm{m}$ thick microbeams and a c-t-c distance of $400 \mu \mathrm{m}$. The three labelled areas correspond to skin (a), skull (b) and brain (c). Statistical uncertainty bars are at two standard deviations.

For this, we relied on the $\mathrm{NTD}_{2.0}$ to establish the equivalence between the valley dose (given in one fraction) with the standard fractionation scheme of $2 \mathrm{~Gy} /$ fraction.

\subsection{Dose distributions for a centrally located tumour}

The most conservative tumour location to evaluate the absorbed dose in the healthy tissue is the centre of the brain. Using a single port irradiation, the optimum ballistics will be given by a lateral irradiation (ear-to-ear axis). With this configuration, in which the tumour centre is at a depth of $7.2 \mathrm{~cm}$ with respect to the skin entrance (Harling et al 1995), the quantity of healthy irradiated tissue is minimized.

Figure 2 shows the ratio between the peak dose and the valley dose, i.e. the PVDR, as a function of depth. The PVDR takes values around 58 in the first $2 \mathrm{~mm}$ (skin), but it falls off rapidly in the bone, reaching a value of 18 in the interface with the brain. This drastic reduction in bone with respect to skin is due to the increase of the fluence of photons that are Compton scattered into the valley region. These photons have a larger probability per unit mass of undergoing a photoelectric absorption in bone since its average atomic number is higher. This results in a higher local dose deposition. In the brain, the PVDR remains almost constant $(\mathrm{PVDR} \simeq 25)$.

Figures 3 and 4 display the valley dose distribution, the peak dose distribution and the $\mathrm{NTD}_{2.0}$ for different dose prescriptions in the centre of the tumour ranging from 40 to $65 \mathrm{~Gy}$ in one fraction. The maximum valley doses are $2-5 \mathrm{~Gy}$ in the skin, 9-17 Gy in the bone and $4-8 \mathrm{~Gy}$ in the brain. The maximum values of $\mathrm{NTD}_{2.0}$ are $2-6 \mathrm{~Gy}$ in the skin, $30-80 \mathrm{~Gy}$ in the bone and 6-18 Gy in the brain. Owing to the high effective atomic number of the bone, the skull receives a substantial dose and it will be the limiting organ to establish the maximum peak dose in the tumour.

The tolerance for the bone lies between 50 and $65 \mathrm{~Gy}$ for a $5 \%$ probability of complications within 5 years (Emami et al 1991). Therefore, from figure 4, the maximum tumour peak dose prescription achievable is $55 \mathrm{~Gy}$ and the corresponding valley dose in the tumour is $2.6 \mathrm{~Gy}$ (see figure 3 left). Considering this and the information displayed in figure 3 , the maximum peak and valley doses for all the tissues can be established. The maximum skin entrance dose is $200 \mathrm{~Gy}$ and the maximum peak doses in the skull and the brain are 315 and $150 \mathrm{~Gy}$. The corresponding valley doses in the skin, skull and brain are 4, 14 and $7 \mathrm{~Gy}$, respectively. In 

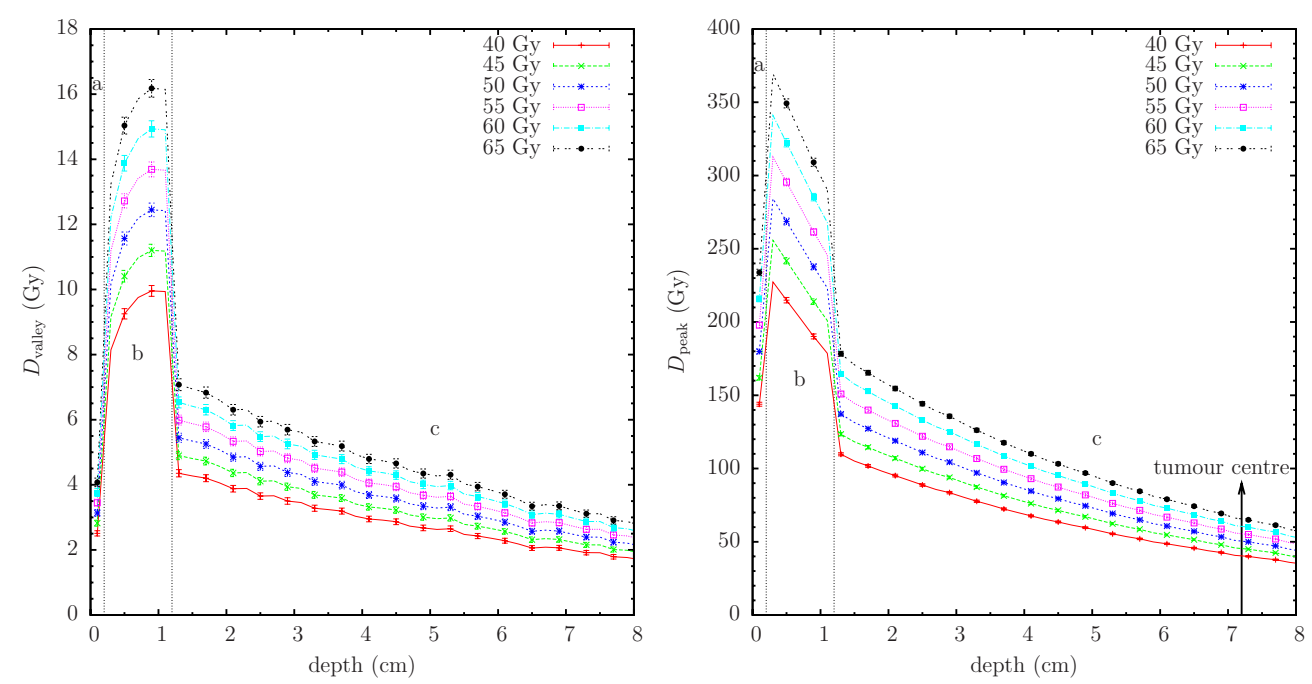

Figure 3. Valley dose (left) and peak dose (right) distributions for skin (a), skull (b) and brain (c) for the indicated peak dose prescriptions in the tumour, situated at a depth of $7.2 \mathrm{~cm}$. The c-t-c distance between the $50 \mu \mathrm{m}$ thick microbeams is $400 \mu \mathrm{m}$. Each curve is proportional to the tumour dose prescription.

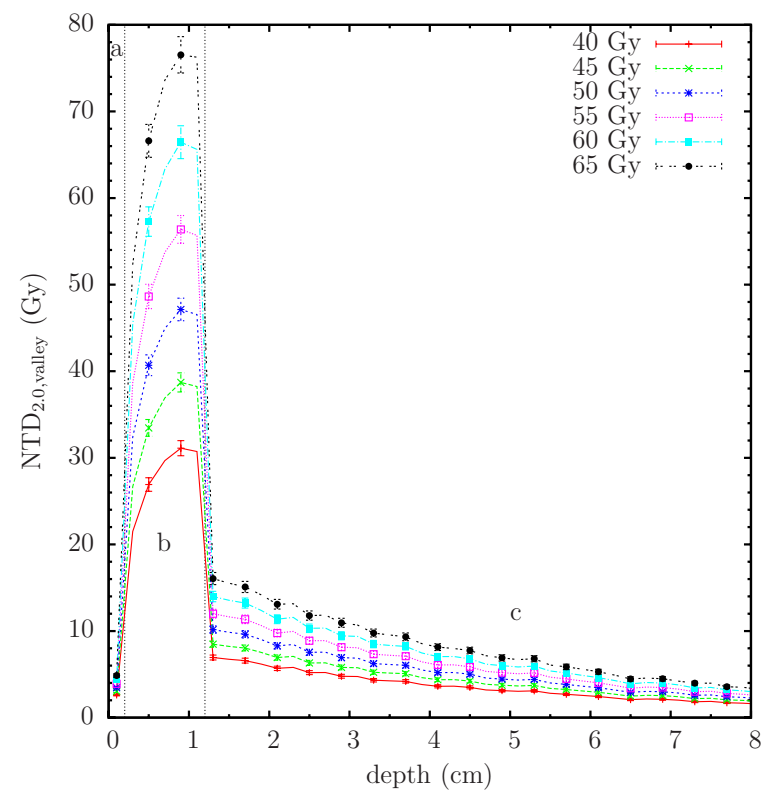

Figure 4. $\mathrm{NTD}_{2.0}$ valley dose distribution for the different tissues: skin (a), skull (b) and brain (c) for the indicated peak dose prescriptions in the tumour, situated at a depth of $7.2 \mathrm{~cm}$. The c-t-c distance between the $50 \mu \mathrm{m}$ thick microbeams is $400 \mu \mathrm{m}$.

summary, if the peak dose prescription in the tumour is smaller than $55 \mathrm{~Gy}$, the valley dose in the healthy tissue will be kept under tolerances. If peak doses of $62 \mathrm{~Gy}$ were delivered to a centrally located tumour, the $50 \%$ probability of bone necrosis within 5 years in bone would be reached (see figure 4). 

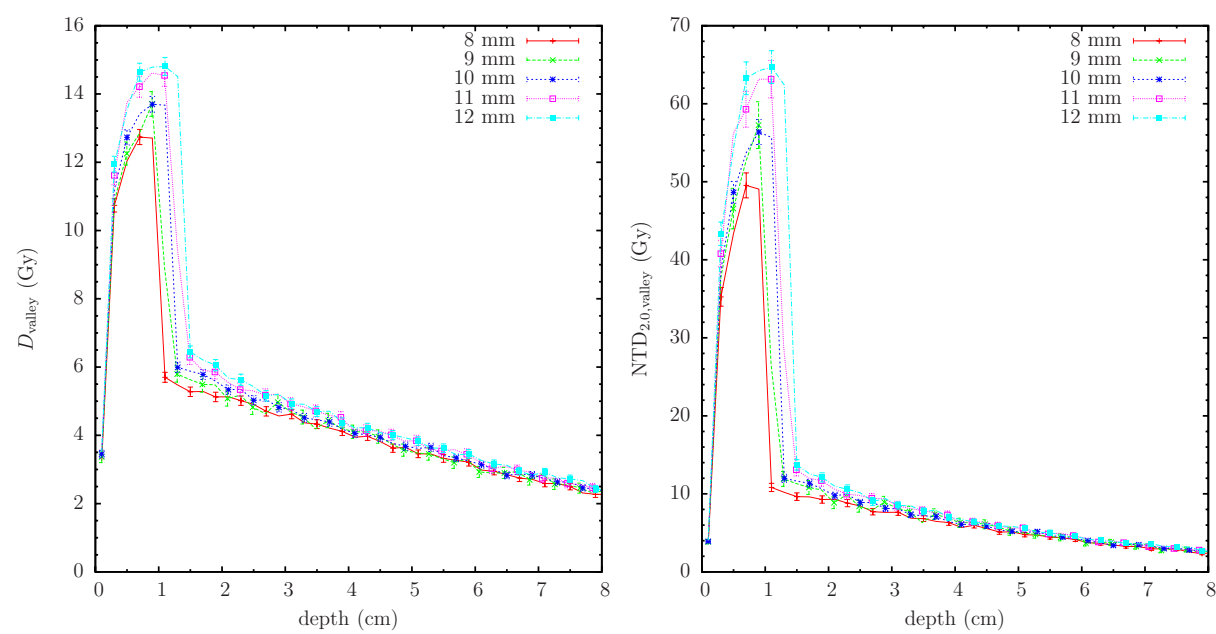

Figure 5. Absorbed dose (left) and $\mathrm{NTD}_{2.0}$ (right) valley dose distributions for skull thicknesses ranging from 8 to $12 \mathrm{~mm}$. The prescribed peak dose in the centre of the tumour, situated at a depth of $7.2 \mathrm{~cm}$, is $55 \mathrm{~Gy}$ in one fraction. The c-t-c distance between the $50 \mu \mathrm{m}$ thick microbeams is $400 \mu \mathrm{m}$.

Table 1. Maximum peak dose prescription and valley dose in the tumour in one fraction keeping the healthy tissue under tolerances ( $5 \%$ complication probability within 5 years) for different tumour positions.

\begin{tabular}{lll}
\hline $\begin{array}{l}\text { Depth of } \\
\text { tumour }(\mathrm{cm})\end{array}$ & $\begin{array}{l}\text { Maximum peak } \\
\text { dose }(\mathrm{Gy})\end{array}$ & $\begin{array}{l}\text { Corresponding } \\
\text { valley dose }(\mathrm{Gy})\end{array}$ \\
\hline 1.5 & 145 & 5.9 \\
2.5 & 125 & 5.2 \\
3.5 & 105 & 4.5 \\
4.5 & 90 & 4.1 \\
5.5 & 75 & 3.5 \\
6.5 & 65 & 2.9 \\
7.2 & 55 & 2.6 \\
\hline
\end{tabular}

\subsection{Dose distributions as a function of tumour position}

The valley doses and the corresponding $\mathrm{NTD}_{2.0}$ values have been calculated for a number of tumour positions with respect to the head surface. Table 1 lists, for several tumour locations, the maximum peak dose that can be prescribed in the tumour to keep the corresponding valley doses below healthy tissue tolerances. As can be seen, a deeper-seated tumour implies a lower maximum peak dose prescription to fulfil the requested condition.

\subsection{Dose distributions as a function of bone thickness}

The above calculations have been done for a skull thickness of $10 \mathrm{~mm}$. However, there might be some slight variation among individuals. Figure 5 shows the valley and NTD $_{2.0}$ dose distributions for several skull thicknesses close to the value used in this work $(8,9,11$ and $12 \mathrm{~mm}$ ). Doses were obtained for a tumour dose prescription of $55 \mathrm{~Gy}$ in one fraction. There are appreciable differences in the bone but there is no variation of the deposited dose as a function of depth (beyond $2 \mathrm{~cm}$ ). It is important to point out that the difference in $\mathrm{NTD}_{2.0}$ between considering $8 \mathrm{~mm}$ or $12 \mathrm{~mm}$ of skull is about $15 \mathrm{~Gy}$. As a consequence, one should prescribe doses that are several Gy below the tissue tolerances to account for these variations. 


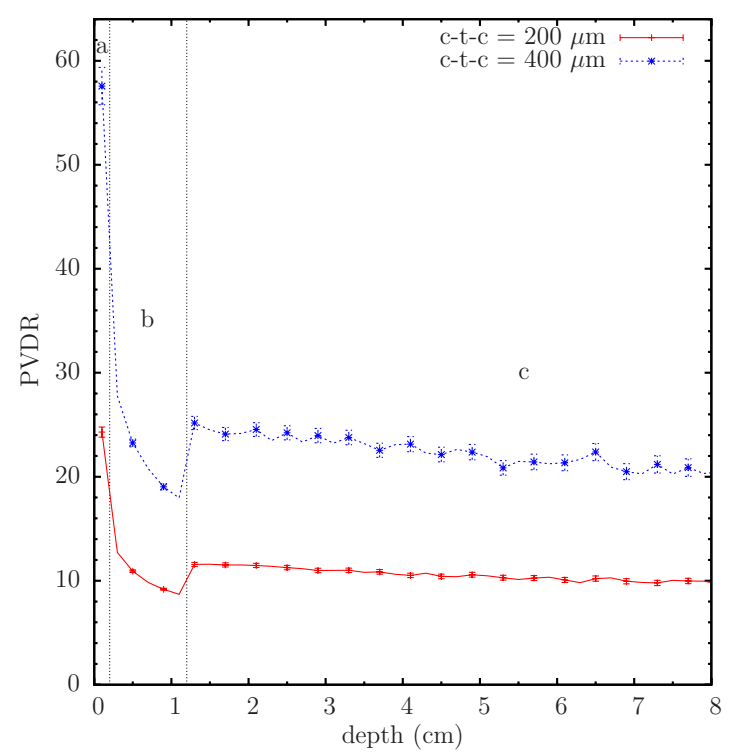

Figure 6. PVDR as a function of depth for two separations between microbeams. The dashed and solid curves correspond to c-t-c separations of 400 and $200 \mu \mathrm{m}$, respectively.

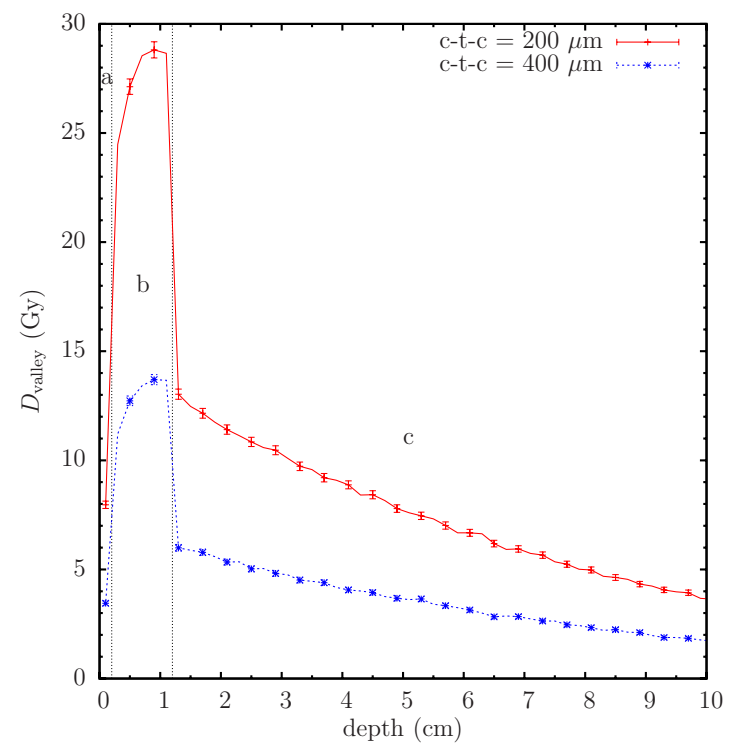

Figure 7. Valley dose distribution as a function of depth for two separations between the microbeams. The dashed and solid curves correspond to c-t-c separations of 400 and $200 \mu \mathrm{m}$, respectively. The prescribed peak dose in the centre of the tumour, situated at a depth of $7.2 \mathrm{~cm}$, is $55 \mathrm{~Gy}$ in one fraction.

\subsection{Dose distributions as a function of the separation between microbeams}

Figure 6 compares the PVDR values for two c-t-c distances between microbeams, namely 200 and $400 \mu \mathrm{m}$. The PVDR for a c-t-c distance of $200 \mu \mathrm{m}$ is lower because of the higher valley dose. Figure 7 presents the valley dose distribution corresponding to a peak dose prescription 
of 55 Gy in the centre of the tumour. With a $200 \mu \mathrm{m}$ c-t-c distance, it is possible to deliver a maximum dose of only $27 \mathrm{~Gy}$ in the tumour, maintaining the healthy tissue under tolerances, i.e. half than that for a c-t-c separation of $400 \mu \mathrm{m}$. The valley dose in a centrally located tumour is now $3.3 \mathrm{~Gy}$. Therefore, to scale up the doses with respect to the ones in conventional radiosurgery, a c-t-c distance of $400 \mu \mathrm{m}$ is recommended.

\section{Conclusions}

MC simulations in a human head phantom have been performed to define safe irradiation protocols for the forthcoming clinical trials in MRT. The aim of these trials is the quest for a radical treatment of gliomas, which are the most aggressive and radioresistant brain tumours. The results of this work show that it is possible to deliver a relatively large dose to the tumour and, at the same time, keep the valley dose in the healthy tissue under tolerances. In order to establish the equivalence of the valley dose (one fraction) with the standard fractionation scheme, the $\mathrm{NTD}_{2.0}$ has been used. The maximum peak dose that can be prescribed to the tumour while sparing the surrounding normal tissue has been evaluated for various tumour positions. The skull is the organ with the largest absorbed doses. For the most conservative tumour location, i.e. in the centre of the brain, the maximum peak and valley doses accomplishable in the tumour are $55 \mathrm{~Gy}$ and $2.6 \mathrm{~Gy}$, respectively, in one fraction and with a unidirectional irradiation. In this way, the valley dose in the healthy tissue will remain under tolerances (5\% probability of complication within 5 years). A level of $50 \%$ probability of bone necrosis would be reached if the tumour were irradiated with a peak dose of $62 \mathrm{~Gy}$. Although there is no data on the doses required to ablate glioma tumours in humans using MRT, existing data from conventional radiotherapy indicate that an aggressive treatment would enhance the patient lifespan.

\section{Acknowledgments}

The authors would like to thank the support given by the ESRF, in particular the help of Rainer Wilcke of the Scientific Software Group. JS acknowledges financial support from the Spanish Ministerio de Educación y Ciencia (projects no. FIS2006-07016 and FPA2009-14091-C02-01) and from the Spanish networking research center CIBER-BBN. JMFV acknowledges financial support from the Spanish Ministerio de Educación y Ciencia (projects no. FPA2006-12066 and FPA2009-14091-C02-01) and FEDER.

\section{References}

Barth R F, Coderre J A, Vicente M G H and Blue T E 2005 Boron neutron capture therapy of cancer: current status and future prospects Clin. Cancer Res. 11 3987-4002

Behin A, Hoang-Xuan K, Carpentier A F and Delattre J Y 2003 Primary brain tumours in adults Lancet Oncol. $361323-31$

Blattmann H et al 2005 Applications of synchrotron x-rays for radiotherapy Nucl. Instrum. Methods A 548 17-22

Boudou C, Balosso J, Estève F and Elleaume H 2005 Monte Carlo dosimetry for synchrotron stereotactic radiotherapy of brain tumours Phys. Med. Biol. 50 4841-51

Bräuer-Krisch E, Requardt H, Brochard T, Berruyer G, Renier M, Laissue J A and Bravin A 2009 New technology enables high precision multislit collimators for microbeam radiation therapy Rev. Sci. Instrum. 80074301

Brenner D J 2008 The linear-quadratic model is an appropriate methodology for determining isoeffective doses at large doses per fraction Semin. Radiat. Oncol. 18 234-9 
Cardinale R M, Benedict S H, Wu Q, Zwicker R D, Gaballa H E and Mohan R 1998 A comparison of three stereotactic radiotherapy techniques: ARCS vs. noncoplanar fixed fields vs. intensity modulation Int. J. Radiat. Oncol. Biol. Phys. 42 431-6

Company F Z and Allen B J 1998 Calculation of microplanar beam dose profiles in a tissue/lung/tissue phantom Med. Phys. Biol. 43 2491-501

Cullen D E, Hubbell J H and Kissel L 1997 EPDL97: The evaluated photon data library, 97 version. Report UCRL-50400 vol 6 (Livermore, CA: Lawrence Livermore National Laboratory)

Curtis H J 1967 The use of a deuteron microbeam for simulating the biological effects of heavy cosmic-ray particles Radiat. Res. Suppl. 7 250-7

De Felici M, Felici R, Sanchez del Rio M, Ferrero C, Bacarian T and Dilmanian F A 2005 Dose distributions from X-ray microbeam arrays applied to radiation therapy: an EGS4 Monte Carlo study Med. Phys. 32 2455-63

Dilmanian F A et al 2001 Response of avian embryonic brain to spatially segmented x-ray microbeams Cell. Mol. Biol. (Noisy-le-grand) 47 485-93

Dilmanian F A et al 2002 Response of rat intracranial 9 L gliosarcoma to microbeam radiation therapy Neuro Oncol. 4 26-38

Dilmanian F A, Morris G M, Zhong N, Bacarian T, Hainfeld J F, Kalef-Ezra J, Brewington L J, Tammam J and Rosen E M 2003 Murine EMT-6 carcinoma: high therapeutic efficacy of microbeam radiation therapy Radiat. Res. $159632-41$

Dilmanian F A, Zhong Z, Bacarian T, Benveniste H, Romanelli P, Wang R, Welwart J, Yuasa T, Rosen E M and Anschel D J 2006 Interlaced X-ray microplanar beams: a radiosurgery approach with clinical potential Proc. Natl Acad. Sci. USA 103 9709-14

Emami B, Lyman J, Brown A, Coia L, Goitein M, Munzenrider J E, Shank B, Solin L J and Wesson M 1991 Tolerance of normal tissue to therapeutic irradiation Int. J. Radiat. Oncol. Biol. Phys. 21 109-22

Fernández-Varea J M, Carrasco P, Panettieri V and Brualla L 2007 Monte Carlo based water/medium stopping-power ratios for various ICRP and ICRU tissues Phys. Med. Biol. 52 6475-83

Flickinger J C and Kalend A 1990 Use of normalized total dose to represent the biological effect of fractionated radiotherapy Radiother. Oncol. 17 339-47

Garcia L M, Leblanc J, Wilkins D and Raaphorst G P 2006 Fitting the linear-quadratic model to detailed data sets for different dose ranges Phys. Med. Biol. 51 2813-23

Guerrero M and Li X A 2004 Extending the linear-quadratic model for large fraction doses pertinent to stereotactic radiotherapy Phys. Med. Biol. 49 4825-35

Harling O K, Roberts K A, Moulin D J and Rogus R D 1995 Head phantoms for neutron capture therapy Med. Phys. 22 579-83

ICRU 371984 Stopping Powers for Electrons and Positrons ICRU Report No 37 (Bethesda, MD: ICRU)

Joiner M and van der Kogel A (ed) 2009 Basic Clinical Radiobiology (London: Edward Arnold)

Kagan A R, Steckel R J, Cancilla P, Juillard G and Patin T 1976 The pathogenesis of brain necrosis: time and dose parameters Int. J. Radiat. Oncol. Biol. Phys. 1 729-32

Kirkpatrick J P, Brenner D J and Orton C G 2009 POINT/COUNTERPOINT: the linear-quadratic model is inappropriate to model high dose per fraction effects in radiosurgery Med. Phys. 36 3381-4

Laissue J A et al 1998 Neuropathology of ablation of rat gliosarcomas and contiguous brain tissues using a microplanar beam of synchrotron-wiggler-generated X rays Int. J. Cancer 78 654-60

Laissue J A et al 1999 Microbeam radiation therapy Proc. SPIE 3770 38-45

Laissue J A et al 2001 The weanling piglet cerebellum: a surrogate for tolerance to MRT (microbeam radiation therapy) in pediatric neuro-oncology Proc. SPIE 4508 65-73

Laissue J A, Blattmann H, Wagner H P, Grotzer M A and Slatkin D N 2007 Prospects for microbeam radiation therapy of brain tumours in children to reduce neurological sequelae Dev. Med. Child. Neurol. 49 577-81

Lawrence Y R, Li X A, el Naqa I, Hahn C A, Marks L B, Merchant T E and Dicker A P 2010 Radiation dose-volume effects in the brain Int. J. Radiat. Oncol. Biol. Phys. 76 S20-7

Lind B K, Persson L M, Edgren M R, Hedlöf I and Brahme A 2003 Repairable-conditionally repairable damage model based on dual Poisson processes Radiat. Res. $160366-75$

Miura M, Blattmann H, Bräuer-Krisch E, Bravin A, Hanson A L, Nawrocky M M, Micca P L, Slatkin D N and Laissue J A 2006 Radiosurgical palliation of aggressive murine SCCVII squamous cell carcinomas using synchrotrongenerated x-ray microbeams Br. J. Radiol. 79 71-5

Orion I, Rosenfeld A B, Dilmanian F A, Telang F, Ren B and Namito Y 2000 Monte Carlo simulation of dose distributions from a synchrotron-produced microplanar beam array using the EGS4 code system Phys. Med. Biol. 45 2497-508 
Overgaard M 1988 Spontaneous radiation-induced rib fractures in breast cancer patients treated with postmastectomy irradiation - a clinical radiobiological analysis of the influence of fraction size and dose-response relationships on late bone damage Acta Oncol. 27 117-22

Phillips M H, Stelzer K J, Griffin T W, Mayberg M R and Winn H R 1994 Stereotactic radiosurgery: A review and comparison of methods J. Clin. Oncol. 12 1085-99

Prezado Y, Fois G, Edouard M, Nemoz C, Renier M, Requardt H, Estève F, Adam J F, Elleaume H and Bravin A 2009a Biological equivalent dose studies for dose escalation in stereotactic synchrotron radiation therapy clinical trials Med. Phys. 36 725-33

Prezado Y, Fois G, Le Duc G and Bravin A 2009b Gadolinium dose enhancement studies in microbeam radiation therapy Med. Phys. 36 3568-74

Regnard P et al 2008 Irradiation of intracerebral 9 L gliosarcoma by a single array of microplanar x-ray beams from a synchrotron: balance between curing and sparing Phys. Med. Biol. 53 861-78

QUANTEC 2010 Quantitative analyses of normal tissue effects in the clinic Int. J. Radiat. Oncol. Biol. Phys. $76 \mathrm{~S} 1-160$

Renier M et al 2008 The radiotherapy clinical trials projects at the ESRF: technical aspects Eur. J. Radiol. 68S S147-50

Sachs R K, Hahnfeld P and Brenner D J 1997 The link between low-LET dose-response relations and the underlying kinetics of damage production repair misrepair Int. J. Radiat. Biol. 72 351-74

Salvat F, Jablonski A and Powell C J 2005 ELSEPA-Dirac partial-wave calculations of elastic scattering of electrons and positrons by atoms, positive ions and molecules Comput. Phys. Commun. 165 157-90

Salvat F, Fernández-Varea J M and Sempau J 2006 PENELOPE-2006, A Code System for Monte Carlo Simulation of Electron and Photon Transport (Issy-les-Moulineaux: OECD Nuclear Energy Agency)

Sempau J, Acosta E, Baró J, Fernández-Varea J M and Salvat F 1997 An algorithm for Monte Carlo simulation of coupled electron-photon transport Nucl. Instrum. Methods B 132 377-90

Sempau J, Sánchez-Reyes A, Salvat F, Oulad ben Tahar H, Jiang S B and Fernández-Varea J M 2001 Monte Carlo simulation of electron beams from an accelerator head using PENELOPE Phys. Med. Biol. 46 1163-86

Sempau J and Andreo P 2006 Configuration of the electron transport algorithm of PENELOPE to simulate ion chambers Phys. Med. Biol. 51 3533-48

Sempau J and Badal A 2008 PenEasy, a modular main program and voxelised geometry package for PENELOPE http://www.upc.edu/inte/downloads/penEasy.htm

Serduc R et al 2008 Characterization and quantification of cerebral edema induced by synchrotron x-ray microbeam radiation therapy Phys. Med. Biol. $531153-66$

Shaw E, Scott C, Souhami L, Dinapoli R, Kline R, Loeffler J and Farnan N 2000 Single dose radiosurgical treatment of recurrent previously irradiated primary brain tumors and brain metastases: Final report of RTOG PROTOCOL 90-05 Int. J. Radiat. Oncol. Biol. Phys. 47 291-8

Siegbahn E A, Stepanek J, Bräuer-Krisch E and Bravin A 2006 Determination of dosimetrical quantities used in microbeam radiation therapy (MRT) with Monte Carlo simulations Med. Phys. 33 3248-59

Slatkin D N, Spanne P, Dilmanian F A and Sandborg M 1992 Microbeam radiation therapy Med. Phys. 19 1395-400

Slatkin D N, Spanne P, Dilmanian F A, Gebbers J O and Laissue J A 1995 Subacute neuropathological effects of microplanar beams of x-rays from a synchrotron wiggler Proc. Natl Acad. Sci. USA 92 8783-7

Smilowitz H M et al 2006 Synergy of gene-mediated immunoprophylaxis and microbeam radiation therapy for advanced intracerebral rat $9 \mathrm{~L}$ gliosarcomas Neuro Oncol. $78135-43$

Spiga J, Siegbahn E A, Bräuer-Krisch E, Randaccio P and Bravin A 2007 The GEANT4 toolkit for microdosimetry calculations: application to microbeam radiation therapy (MRT) Med. Phys. 34 4322-30

Steel G G (ed) 2002 Basic Clinical Radiobiology 3rd edn (London: Edward Arnold)

Stepanek J, Blattmann H, Laissue J A, Lyubimova N, Di Michiel M and Slatkin D N 2000 Physics study of microbeam radiation therapy with PSI-version of Monte Carlo code GEANT as a new computational tool Med. Phys. 27 1664-75

Sterpin E, Salvat F, Cravens R, Ruchala K, Olivera G H and Vynckier S 2008 Monte Carlo simulation of helical tomotherapy with PENELOPE Phys. Med. Biol. 53 2161-80

Stupp R et al 2002 Promising survival for patients with newly diagnosed glioblastoma multiforme treated with concomitant radiation plus temozolomide followed by adjuvant temozolomide J. Clin. Oncol. 20 1375-82

Stupp R et al 2005 Radiotherapy plus concomitant and adjuvant temozolomide for glioblastoma $N$. Engl. J. Med. 352 987-96 
Stupp R et al 2009 Effects of radiotherapy with concomitant and adjuvant temozolomide versus radiotherapy alone on survival in glioblastoma in a randomised phase III study: 5-year analysis of the EORTC-NCIC trial Lancet Oncol. 10 459-66

Torres J, Buades M J, Almansa J F, Guerrero R and Lallena A M 2004 Dosimetry characterization of ${ }^{32} \mathrm{P}$ intravascular brachytherapy source wires using Monte Carlo codes PENELOPE and GEANT4 Med. Phys. 31 296-304

Turesson I and Thames H D 1989 Repair capacity and kinetics of human skin during fractionated radiotherapy: Erythema, desquamation, and telangiectasia after 3 and 5 years follow-up Radiother. Oncol. 15 169-88 